\title{
Impulse Regulation in Anorexia Nervosa and Bulimia Nervosa: Some Formulations
}

\author{
STAFFAN SOHLBERG
}

Associate Professor of Clinical Psychology at Uppsala University, Sweden*

\begin{abstract}
Empirical observations imply that impulsivity is specifically associated with poor prognosis in eating disorders. The present paper cites studies suggesting that this factor is predominantly associated with the bulimic pattern of eating disturbance, while "restriction" may be associated rather with hypercontrol of behaviour. Further evidence is cited suggesting that the relationship between hyper- and hypocontrol is actually very intimate, both on a behavioural and biochemical level. Rather than bulimics being generally impulsive and "restrictors" generally hypercontrolled, future studies may reveal impulse regulation difficulties of both kinds in both groups. Single-minded preoccupation with dieting may be an ill-equipped individual's effort to simplify life in periods of change and environmental challenge that demand capacity for flexible impulse regulation.
\end{abstract}

\section{Introduction}

Contrary to impressions of both professionals and the lay public, recent evidence suggests that the incidence of anorexia nervosa may not be greater today than during previous decades (Lucas et al., 1988; Nielsen, 1990). Still, this disorder and related patterns of weight-shape preoccupation, body image disturbance and severely disordered eating (bulimia nervosa and atypical or subclinical variants) continue to present significant health problems. Thus, beyond the risks directly associated with starvation, binge eating and purging, there are often significant and long-lasting psychological and social sequelae.

Some $90-95 \%$ of patients are women and there is a fair amount of consensus that the disorders begin with dieting, often starting in the context of separation (e.g. going to boarding school, au pair work in another country, loss of a love relationship). For some, dieting escalates into frankly anorexic food refusal and emaciation. For others dieting is interrupted by periods of binge eating in which huge amounts of food are consumed in a short time, only to be purged immediately afterwards. When the afflicted individual eventually seeks help, her condition is a mixture of basic psychopathology and the effects of acute malnutrition (e.g. Woell et al., 1989). No firm conclusions have been drawn concerning why some dieters go on to have clinical eating disorders. In the present paper I will discuss how investigation of impulse regulation could shed some light on this issue.

* At the time of writing this paper Visiting Research Scholar at the University of California Los Angeles Neuropsychiatric Institute.

$0953-4180 / 91 / 030189+13 \$ 3.50 / 0 \quad$ (C) 1991 CNS (Clinical Neuroscience) Publishers 
Themes to be explored include the role of impulsivity for poor long-term outcome, the possibility that hypercontrol of impulses is a trait that is qualitatively different but equally pathological, and the differences and similarities in impulse regulation between "restrictors" and "bulimics".

\section{Impulsivity predicts poor long-term outcome}

The inspiration for this endeavour came through finding impulsivity to be a specific predictor of poor long term outcome, in a mixed sample of anorexics and bulimics (Sohlberg et al., 1989; Diagnostic terms refer to the disorders as defined in the American Psychiatric Association's manuals DSM-III and DSM-III-R). Sohlberg et al. constructed an impulsivity index as a sum score for the presence of binge eating, shoplifting, alcohol/drug abuse, and suicide attempts. Scores from presentation on this index proved to be the best predictor of all when patients were followed-up on the average 2.5 years later, with more impulsive individuals having more disordered eating, as well as being more depressed. They also had lower level of overall mental health.

The authors interpreted their findings as due to impulsivity being a marker for personality disorder. Since personality disordered patients can $a$ priori be expected to come out more poorly on almost any follow-up instrument that measures an aspect of mental health, this interpretation accounted for the broad influence of impulsivity. Sohlberg et al. therefore hypothesized specifically that in a new follow-up of the same sample, impulsivity would predict not only eating disorder, but lower overall mental health as well. Interestingly, after another 2.5 years follow-up they found support for the first part of the prediction but not the latter. Impulsivity still predicted eating disorder, but only had a very weak and nonsignificant relation to overall mental health. This could be a method artefact due to denial of illness in the less impulsive subjects, if illness was easier to deny on the self-rating test that was used to measure eating disorder than on the interview measure of mental health. However, less impulsive subjects did not score lower on the self-report Beck Depression Inventory (Beck et al., 1961), as they might have done if they were anxious to appear to be functioning well. Thus, impulsivity came out as a replicable predictor, specifically tied to chronic eating disorder.

This is a very interesting finding considering how replicable prognostic indicators are rarer in this field than was once believed, let alone specific ones (Herzog et al., 1988; Sohlberg et al., in press; Strober, 1990). At the same time, there is much to suggest that the problem of impulse control in eating disorders would benefit from analysis in a more theoretically thorough way than was the case in this study, and some other previous studies as well.

In a sense, abnormalities of impulse control would seem to be present in these disorders by definition. There is now consensus that preoccupation with weight and shape is an important characteristic of bulimia nervosa (DSM-III-R; Fairburn and Garner, 1986; Holmgren et al., 1983; Russell, 1979), but eating large amounts of food in a state of dyscontrol is the 
hallmark of the disorder and its most easily diagnesed feature. Simultaneously, one of the most common ways to introduce the phenomenology of anorexia nervosa is to tell how the name is a misnomer since patients are not anorexic at all: they are intensely hungry, except if the disorder goes to a very chronic stage when hunger feelings can actually be absent. If scant eating is recognized to occur in the context of severe hunger then, an almost unbelievable amount of control over the most basic of all impulses appears to be present.

Attempts by Sohlberg et al. to explain their findings included absence of impulsivity as representing a "will-power" potential that was absent in less controlled individuals. Citing Björvell et al. (1985) and incidental results reported by Vandereycken and Vanderlinden (1983), they also suggested cognitive disturbances in impulsive individuals. The latter authors found psychological test data patterns suggestive of such disturbances in anorexics who scored highly on the same test used by Sohlberg et al. and the former suggested that inability to learn from experience is a typical cognitive disturbance in an "impulsiveness syndrome".

Both these explanations essentially imply that lax impulse control is unfavourable. However, it is perfectly possible to assume that the true relationship is curvilinear: adaptive functioning requires a balance between expressing and holding back impulses, and too much holding back is no less pathological than too much expression. Heilbrun and Bloomfield (1986) cited several theorists in support of a prediction that college women with "anorexic-like" qualities would score in the hypercontrol range on a measure of self-control, but due to technical problems the results were difficult to interpret. However, Woznica (1990) recently reported results of just this type. Thus, the patients who scored 0 in the Sohlberg et al. study could have included both those whose impulse control was normal or only somewhat too strong, and those whose control was much too strong. In order for this difference to be well represented, observation of various additional behaviours would have been advantageous (e.g. extreme discomfort at minor rule-breaking, fear of taking even prescribed drugs or undergoing medically necessary surgical procedures because this entails having less control over what happens to the body, inability to express even strong feelings and opinions, spending extremely small amounts of money for own purposes, alcohol phobia). If this had been done, more outcome variance could perhaps have been explained in finding the poorest outcomes not only among those who were the most impulsive, but also the least.

Obsessive-compulsive traits, as seen in emotional constraint, and perfectionism are well-known concomitants of anorexia nervosa (Swift et al., 1986). Garfinkel and Garner (1982, cited in Holden, 1990) warned that this could actually be an illusory association, if these traits cause a poorer prognosis and made the patients who have them over-represented in clinical studies. However, a review in preparation seems to suggest that this process cannot explain the data (Sohlberg and Strober, in prep.). Starvation is known to affect both moods and behaviour, but it does not seem satisfactory to explain the observations as due to starvation either, as several studies that depict anorexics as obsessional tested patients only after weight recovery. 
Indeed, 50-year-old suggestions that anorexia nervosa is a form of obsessivecompulsive illness have recently been revived (Rothenberg, 1990) and disputed (Holden, 1990). Importantly however, even the latter author conceded that obsessive-compulsive traits are a part of the premorbid picture.

Impulsivity has been associated with bulimia nervosa (Pyle et al., 1981) and the bulimic subgroup in anorexia nervosa (e.g. Strober, 1981). The latter group resemble classic "restricting" cases (the subgroup who lose weight only by abstaining from eating), but indulge in binge eating and vomit or use other means of compensating for caloric intake. These bulimic anorexics on the whole seem to have more in common with normal-weight bulimics than with restricting anorexics (Garner et al., 1985; Norring, et al., 1989). If emphasis is put on the emotionally constrained and cognitively rigid aspects of obsessive-compulsive traits, this suggests a very straightforward model: restrictive eating is part of a generalized hypercontrol syndrome and bulimic eating of one of hypocontrol. As reported by Logan and Cowan (1984; cited in Schachar and Logan, 1990), "... research ... indicates remarkable consistency in the degree of control across a range of motor, speech, and cognitive tasks" (p. 719).

Direct evidence for this model came recently in the above-mentioned study by Woznica (1990). The study's primary instrument was a comprehensive measure assessing both behavioural and cognitive aspects of impulse control (the Self-Report Test of Impulse Control). Restrictors scored higher than normals on this instrument, while bulimics scored lower. The findings gain in interest in the light of the fact that the samples were less selected than is often the case in eating disorder studies, with half of each patient group being outpatients and half recruited from self-help groups. Also, normal controls were screened for the absence of psychiatric disorder. There was also a limitation in that while all were bona fide patients in terms of being in some form of treatment, subgroup classification was done via a questionnaire.

The findings by Wonderlich et al. (1990) could perhaps be aligned with Woznica's results, in that these authors reported obsessive compulsive personality disorder (OCPD) in all $60 \%$ of restrictors. OCPD prevalence in bulimics was $0 \%$. Further evidence, though indirect, that points in the same direction was provided by Piran et al. (1988). These authors looked at personality disorders ad modum DSM-III and contrasted the more controlled "anxious" cluster C personality disorders with the less controlled "dramatic-erratic" cluster B disorders. The former includes the avoidant, dependent, passive-aggressive and compulsive personality disorders, and the second the histrionic, narcissistic, borderline and antisocial. Sixty-six per cent of bulimics had dramatic-erratic disorders versus $0 \%$ among the restrictors. Conversely, anxious diagnoses were much more common among the restrictors $(77 \%$ vs $29 \%)$.

Several recent studies add to previous ones in emphasizing the greater impulsivity of bulimics. Toner et al. (1987) reported that formerly bulimic anorexics made twice as many errors on a test for impulsivity (the Matching 
Familiar Figures Test) as former restricting anorexics. de Silva and Eysenck (1987) found normal-weight bulimics to score higher than restricting anorexics on psychoticism on the Eysenck Personality Questionnaire (EPQ). Impulsivity is a prominent feature underlying high scores in this scale. Also, Piran et al. (1988) reported greater impulsivity on the Diagnostic Interview for Borderlines in bulimics than in restrictors.

Thus, there are data to support the inference that bulimics are more impulsive than restrictors, and restrictors may even be more controlled than normals. Possible neurobiological differences have been explored but this is difficult because of confounding effects of malnutrition and weight loss (Fava et al., 1989). However, some interesting data refer to serotonin and monoamine oxidase. Soubrié (1986) integrated a large number of animal and human data in arguing that serotonin plays a role of inhibiting action, and Murphy et al. (1989) cited two studies that reported 20-30\% higher concentrations of the serotonin metabolite 5HIAA in Obsessive Compulsive Disorder patients than in normals, while "... impulsive ... patients who are phenomenologically opposite to those with OCD, have 5HIAA levels that are significantly lower ..." (p. 19). ${ }^{1}$ Fava et al. (1989) cited animal studies showing that the inhibitory role of serotonin includes curtailing eating, to make consideration of this substance directly relevant to eating disorders. In fact, links have been made in that Kaye et al. found bulimic patients who binged to the point of satiation have an increase in their plasma Ltryptophan-large neutral amino acids ratio, whereas those who were not satiated by bingeing did not (1988, cited in Fava et al., 1989). Since the increased competitiveness of tryptophan would have led to increased serotonin synthesis, this suggests that increased levels of serotonin were necessary for fullness and inhibition of eating. Kaye et al., (1984a) reported that the serotonin-level of weight-recovered anorexics was not different from that of controls. However, when the sample was divided into restrictors and bulimics, the former had increased serotonin metabolism (Kaye et al., 1984b).

The enzyme monoamine oxidase (MAO) has received attention in eating disorders primarily in the context of pharmacological treatment of depression. Low levels of MAO are firmly linked with impulsivity in normals and in conditions other than eating disorders (Schalling et al., 1988). A recent study by Hallman et al. (1990) reported low levels in weight recovered bulimics. Even more intriguing, Schalling et al. (1988) stated that abnormal MAO levels are associated with "suspiciousness". It has been suggested clinically that eating disordered individuals have difficulty trusting other people, and this has been substantiated using a frequently used assessment instrument (the Eating Disorder Inventory, Garner and Olmsted, 1984) that specifically includes a scale for this dimension (the subscale for "Interpersonal Distrust"). A suggestion that this variable may deserve increasing attention was recently obtained by our group, as higher levels of

${ }^{1}$ There is no one-to-one correspondence between OCD and obsessive compulsive traits. However, the former is one of the known complications of obsessive compulsive personality disorder (APA, 1987). 
distrust was one of very few potential predictors that appeared robust in a three-year follow-up study (Sohlberg et al., in press).

The significance of Schalling's summary however, lies in that there is a qualitative distinction between the correlates of high and low levels. Low levels, as reported by Hallman et al. in bulimics, are associated predominantly with suspiciousness in "antisocial disorders", while high levels indicate suspiciousness in "paranoid-schizoid disorders". The former disorders could be seen to express hypocontrol of impulses and the latter hypercontrol. Thus, speculatively one can contrast restriction / high MAO / "hypercontrolled" (paranoid) suspiciousness against bulimia / low MAO / "hypocontrolled" (antisocial) suspiciousness.

\section{More complex models}

Some lines of evidence thus associate impulsivity more with bulimics than restrictors, and even suggest neurobiological correlates for this difference. Most likely however, more complex models are needed to accurately depict impulse regulation in these disorders. This does not only refer to interaction between neurotransmitters and neuromodulators making realistic models more complex than those that associate a particular behaviour with stably abnormal levels of one substance, but to the basic data as well. An embarrassment to all straightforward models that contrast restrictors with bulimics is the fact that a substantial minority of patients change from one mode to the other, sometimes even more than once (Holmgren et al., 1983). Other facts indicate a close relationship between restriction and bulimia as well. Whilst the study by Piran et al. (1988) suggested an "anxious" vs "dramatic-erratic" difference between restrictors and bulimics, it was the only one of recent major personality-disorder studies to rely on unstructured interviews. Two that used specifically developed personality disorder interviews did not find this pattern (Gartner et al., 1989, and Wonderlich et al., 1990 who had specifically predicted it). Instead, the former group recorded "anxious" disorders as being the most common in both groups, and the latter reported that dramatic-erratic disorders were equally common in both. (However, as stated above, the Wonderlich study reported a huge difference on one specific disorder, the obsessive-compulsive. This is true even when bulimics are studied irrespective of weight, i.e. including normalweight bulimics, when the proportion only goes up from $0 \%-v s 60$ in restrictors-to $6 \%$ ).

In the study by Woznica (1990), restrictors scored higher than normals on the Self-Report Test of Impulse Control and bulimics lower. However, bulimics still reported firmer impulse control than patients with depression or generalized anxiety who had been screened for the absence of eating disorder. Also, not only restrictors but bulimics too consumed less alcohol than normals. These findings suggest that unqualified characterization of bulimics as impulsive is difficult to justify.

The results by Toner et al. (1987) can be construed similarly. Stating that 
previous authors only demonstrated greater behavioural impulsivity in bulimic anorexics, these authors conducted the first study of cognitive impulsivity in using the Matching Familiar Figures Test (MFFT) to compare former bulimic anorexics with former restricting anorexics. Both number of errors in identifying familiar figures and response latency are thought to indicate impulsivity on the MFFT (Schachar and Logan, 1990). However, Toner and her colleages cited previous work as suggesting only the former indicates cognitive impulsivity. This would seem to support the intuitive impression that response latency is more closely related to behavioural impulsivity, and thus more likely to reveal group differences given previous findings. However, groups did not have dissimilar response latencies.

Neely Wold (1983) was unable to confirm more impulsivity in bulimics than in restrictors on the KDS-3A. This is a little-known test that was nevertheless reported to be well-validated. Samples were small but that did not account for the result as scores were actually higher for the restrictors. Further, Feldman and Eysenck (1986, cited in Shaye, 1989) did not find more impulsivity in normal-weight bulimics than in normals. de Silva and Eysenck (1987) did report normal-weight bulimics to score higher on EPQ impulsiveness (psychoticism) than normals, in contrast to restricting anorexics. However, restrictors also had significantly higher lie scores than bulimics, suggesting that they attempted to minimize responses that produce an undesirable image.

Some important generalizations seem to be implied by these results. First, whilst bulimics are often found to be more impulsive than restrictors, this does not apply to all aspects of impulsivity (Toner) or all studies (Neely Wold). Second, when bulimics are found to be more impulsive than restrictors, they may still be less impulsive than depressed and anxious psychiatric controls (Woznica), or not different from normals (Toner et al.). Third, when differences are recorded, there is a risk they could sometimes be inflated due to restrictors trying to minimize pathology (de Silva and Eysenck).

Relevant to the latter point, Eckert et al. (1987) found that several apparent personality differences between restrictors and bulimics disappeared after a month of inpatient weight restoration. They speculated that some that have been reported in the literature might be due to symptoms of acute illness, or simply to a tendency for bulimics to complain more about such symptoms. A greater tendency for restrictors than bulimics to want to appear controlled could, for example, contribute to both their higher-than-normals scores in Woznica's study of impulse control, and the lower-than-bulimics result in de Silva's and Eysenck's of impulsivity.

Several other explanations for the uneven set of results are possible. Differences might pertain only to some aspects of impulse control and not others. Perhaps in some cases restrictors and bulimics are equally cognitively impulsive, but respond in different ways behaviourally (i.e. rather than going on a binge when tension is too high, the restrictor immediately resolves never ever again to eat even the little she ate today). Or, when they are equally impulsive at some task, they are equal for different reasons (e.g. lack 
of cognitive restraint vs poor internal scanning, Heilbrun and Bloomfield, 1986).

Schachar and Logan (1990) in particular emphasized that there are several psychological processes behind behavioural impulse control. In addition to cognitive impulse control these include inter alia search strategies, decision criteria, and attentional capacity. Their comment that mean reaction time can reflect strategy or attentional capacity, rather than inhibitory control, provides an interesting perspective on the absence of response latency differences in the Toner et al. study. Perhaps comparable response latencies were due to greater inhibition but poorer attentional capacity in restrictors and bulimics vs normals? If so, the net result would have been the same for all groups, even though the underlying processes were vastly different.

\section{Do both restrictors and bulimics have impulse regulation problems?}

Pending clarification of these possibilities, it could perhaps prove useful to regard both groups as wrestling with impulse regulation problems. In the restrictor this preferentially results in a picture of obsessive compulsive traits, while the bulimic preferentially appears to be impulsive. Perhaps the bulimic's problem could be hypothesized to be obsessional hypercontrol that, in contrast to that of the restrictor, is too brittle to remain in force indefinitely. This model could seem to bear some resemblance to Cloninger's (1987a) description of "Type I" alcoholics (referenced in Guerrera, 1990). According to Guerrera, Cloninger characterizes these individuals as having "... periods of abstinence interrupted by drinking binges, guilt and fear about dependence on alcohol, emotional dependence, rigidity, perfectionism and introversion." (p. 559; see also Lacey and Moureli, 1986). The restrictor, of course, would be the absolutist. In both cases control is the primary concern and suggests underlying incapacity to regulate impulses flexibly.

In normals, this hypothesized incapacity to regulate impulses flexibly is illustrated by Sanfuentes and Lolas (1988). These researchers found a correlation between dietary restraint on the Three-Factor Eating Questionnaire and psychoticism on the EPQ. Since impulsivity is a prominent feature underlying high scores in the EPQ Psychoticism scale, this indicates the simultaneous presence of hyper- and hypocontrol. Of particular interest knowing that $90-95 \%$ of eating disorder-patients are women, the pattern was confined to the female subsample. Similarly, Southwick and Steele (1987) reported "consummatory restrainers" (considering eating and drinking restraint together; both variables loaded equally strongly on the canonical variate) to score high on several factors of impulsiveness, including Narrow Impulsiveness (acting without regard for consequences), Nonplanning (preferring spontaneous to planned activity), and Liveliness (rapid thought and decision making). They suggested that "... restraint may be a response to perceptions of poor self-control, and represent a reparative attempt to deal with a self-control deficit" (p. 354). 
Casper (1990) recently stated that "... anecdotal reports and past studies have described quite a range of sometimes contradictory personality characteristics, such as compliance coexisting with obstinacy, obsessivecompulsive features, shyness and introversion along with hysterical traits and impulsivity, or interpersonal sensitivity and stoicism" (p. 156). Her hypothesis was this might at least partly be due to starvation effects on personality measures, and failure to separate restrictors from bulimics. However, it is also possible that these results could to some extent be less conflicting at closer look. The intimate relationship between obsessive and impulsive characteristics is obvious in Schachar and Logan's (1990) description of deficient inhibitory control. They state that deficient inhibitory control is revealed by impulsive behaviours such as failing to correct obviously inappropriate responses. This can be seen to represent impulsivity (the response is not cancelled) that results in an obsessive feature (inordinate perseverance or even an obsessive-compulsive symptom like washing one's hands not once but twenty times).

Thus, the exact nature of the measure and the way the data are interpreted could be crucial. Clearly, a person who is stubborn and obstinate according to one type of observation may be found to be insecure and overly attentive to others' opinions according to another with the former characteristic a self-esteem preserving mechanism compensating for the latter. The importance of this perspective is that it suggests restrictors could appear impulsive and bulimics hypercontrolled, depending on what is measured. Had Sanfuentes and Lolas (1988) and Southwick and Steele (1987) only studied restraint they would have concluded there were some highly restrained subjects in the groups; had they only studied impulsivity they would have concluded the same people were highly impulsive.

\section{Why are there impulse regulation problems in these disorders?}

I would like to conclude with some suggestions as to why restrictors and bulimics have impulse regulation problems, and why the balance globally may be in favour of impulsivity in the bulimic, versus obsessive compulsive traits in the restrictor. These suggestions are speculative but could be heuristically useful. Several studies document abnormal rearing and interaction patterns in the families of eating disordered patients (Strober and Humphrey, 1987). Strober (in press) suggested that this to an important extent is due to extreme combinations of biologically grounded temperaments that are shared by family members. According to him, there are abnormal tendencies to avoid novelty, to condition easily to reward, and to avoid harm. These tendencies do not make for robustness and readiness for challenge (as for instance in the changes associated with puberty), but rather for a sense of vulnerability that is shared by family members and handled largely by avoidance. Case material illustrating the process includes a warm and close-knit family of three in which, following some psychotherapy, the mother and the restrictor daughter revealed their difficulty 
being more open because of fear for the occasional explosive outbursts of the normally "calm" father.

Cloninger (1987b) attempted to link theoretically each of the three temperament dimensions with a particular neurotransmitter: novelty seeking with dopamine, harm avoidance with serotonin, and reward dependence with norepinephrine. However, he also warned that the observable traits depend on how a particular individual is positioned on all three dimensions. For example, an individual who is genetically predisposed to high novelty seeking may appear to be so only if not inhibited by simultaneous high harm avoidance. Perhaps conflict between predispositions, when extreme, is not harmoniously resolved but actually causes vacillation between incompatible behaviour tendencies? Then, the child at risk for an eating disorder might be doubly handicapped: first by innate difficulties with impulse regulation, then by not receiving adequate help from her parents in adapting to these.

Why then would the bulimics globally be more impulsive than restrictors? First, there may be no biological or personality difference at all. Strober (1984) documented higher levels of stressful life changes among those restrictors who crossed over to bulimia, an observation that remained significant even after statistical control for several potential confounds. Impulsive behaviour may simply be the response of any hypercontrolled person exposed to intense stress. Second, five out of six studies found families of bulimics to be more manifestly dysfunctional than those of restrictors (Strober and Humphrey, 1987). Even if stress-levels are similar in restrictor and bulimic families, the higher frequency of acting-out behaviours of parents in these families could serve as a model for the child's behaviour. Third, alcohol problems are known to be more frequent in bulimic families. Though fetal alcohol syndrome has only been implicated in chronic cases of eating disorder (Bakan et al., in press), subtle neuropathies resulting from excessive maternal alcohol use could perhaps set the stage for more indiscriminate impulse expression in some bulimics.

According to Bemis Vitousek and Hollon (1990), "anorexic and bulimic individuals describe feeling overwhelmed by the multiplicity of expectations, responsibilities, and choices that impinge upon them, as on all other participants in contemporary society". Asked to describe the "cognitive essence" of their disorder, every single one among a group of former and actively ill anorexics and bulimics stated a major part to be "simplification of life" (p. 206). Perhaps single-minded focus on dieting is a very easy, albeit eventually dangerous, way to simplify life for a female on her way towards an adulthood that requires a capacity for self-regulation that she does not have; this in a culture in which thinness is associated with beauty and success, and is a major concern among girls already in the early teens (Wardle and Marsland, 1990).

In conclusion, I suggest that while bulimics are more impulsive than restrictors, both groups could usefully be seen as having disturbances in impulse regulation. Future research may make progress by investigating the various psychological processes that account for control in overt behaviour, and needs to account for both hyper- and hypocontrol in contrasting groups with one another, as well as with individuals who do not have eating 
disorders. Perhaps those dieters who have prominent impulse regulation problems are those who are at increased risk to develop an eating disorder, and the greater the difficulties, the greater the treatment needs (Lacey and Evans, 1986) and the risk for the disorder to become chronic (Sohlberg et al., 1989).

\section{Acknowledgement}

Financial support from the Sasakawa Young Leaders Fellowship Fund is gratefully acknowledged.

\section{References}

A. P. A. (1987). Diagnostic and Statistical Manual of Mental Disorders. Third Edition Revised (DSM-III-R) (3rd revised ed.). APA, Washington, D.C.

Bakan, R., Laird Birmingham, C. and Goldner, E. M. (in press). Chronicity in anorexia nervosa: pregnancy and birth complications. International Fournal of Eating Disorders.

Beck, A. T., Ward, C. H., Mendelson, M., Mock, J. and Erbaugh, J. (1961). An inventory for measuring depression. Archives of General Psychiatry, 4, 561-571.

Bemis Vitousek, K. and Hollon, S. D. (1990). The investigation of schematic content and processing in eating disorders. Cognitive Therapy and Research, 14(2), 191-214.

Björvell, H., Edman, G., Rössner, S. and Schalling, D. (1985). Personality traits in a group of severely obese patients. International Fournal of Obesity, 9, 257-266.

Casper, R. C. (1990). Personality features of women with good outcome from restricting anorexia nervosa. Psychosomatic Medicine, 52, 156-170.

Cloninger, C. R. (1987a). Neurogenetic adaptive mechanisms in alcoholism. Science, 236, $410-416$.

Cloninger, C. R. (1987b). A systematic method for clinical description and classification of personality variants: A proposal. Archives of General Psychiatry, 44, 573-588.

de Silva, P. and Eysenck, S. (1987). Personality and addictiveness in anorexic and bulimic patients. Personality and Individual Differences, 8(5), 749-751.

Eckert, E., Halmi, K., Marchi, P. and Cohen, J. (1987). Comparison of bulimic and nonbulimic anorexia nervosa patients during treatment. Psychological Medicine, 17, 891-898.

Fairburn, C. G. and Garner, D. M. (1986). The diagnosis of bulimia nervosa. International Journal of Eating Disorders, 5(3), 403-419.

Fava, M., Copeland, P. M., Schweiger, U. and Herzog, D. B. (1989). Neurochemical abnormalities of anorexia nervosa and bulimia nervosa. American Fournal of Psychiatry, 146(8), 963-971.

Feldman, J. and Eysenck, S. B. (1986). Addictive personality traits in bulimic patients. Personality and Individual Differences, 7, 923-926.

Garfinkel, P. E. and Garner, D. M. (Ed.). (1982). "Anorexia Nervosa. A Multidimensional Perspective" Brunner/Mazel, New York.

Garner, D. M., Garfinkel, P. E. and O'Shaughnessy, M. (1985). The validity of the distinction between bulimia with and without anorexia nervosa. American fournal of Psychiatry, 142(5), 581-587.

Garner, D. M. and Olmsted, M. P. (1984). "Manual for Eating Disorder Inventory (EDI)" Psychological Assessment Resources, Inc., Odessa.

Gartner, A. F., Marcus, R. N., Halmi, K. and Loranger, A. W. (1989). DSM-III-R personality disorders in patients with eating disorders. American Fournal of Psychiatry, 146, $1585-1591$.

Guerrera, R. J. (1990). Some biological and behavioural features associated with clinical personality types. Journal of Nervous and Mental Diseases, 178(9), 556-566.

Hallman, J., Sakurai, E. and Oreland, L. (1990). Blood platelet monoamine oxidase activity, 
serotonin uptake and release rates in anorexia and bulimia patients and in healthy controls. Acta Psychiatrica Scandinavica, 81(1), 73-77.

Heilbrun, A. B. and Bloomfield, D. L. (1986). Cognitive differences between bulimic and anorexic females-Self-control deficits in bulimia. International Fournal of Eating Disorders, $5(2), 209-222$.

Herzog, D. B., Keller, M. B. and Lavori, P. W. (1988). Outcome in anorexia nervosa and bulimia nervosa: A review of the literature. Fournal of Nervous and Mental Diseases, 176(3), 131-143.

Holden, N. L. (1990). Is anorexia nervosa an obsessive-compulsive disorder? British Fournal of Psychiatry, 157, 1-5.

Holmgren, S., Humble, K., Norring, G., Roos, B.-E., Rosmark, B. and Sohlberg, S. (1983). The anorectic bulimic conflict - an alternative diagnostic approach to anorexia nervosa and bulimia. International Fournal of Eating Disorders, 2(2), 3-14.

Kaye, W. H., Ebert, M. H., Raleigh, M. and Lake, R. (1984a). Abnormalities in CNS monoamine metabolism in anorexia nervosa. Archives of General Psychiatry, 41(4), 350355.

Kaye, W. H., Ebert, M. H., Gwirtsman, H. E. and Weiss, S. R. (1984b). Differences in brain serotonergic metabolism between nonbulimic and bulimic patients with anorexia nervosa. American Journal of Psychiatry, 141(12), 1598-1601

Kaye, W. H., Gwirtsman, H. E. and Brewerton, T. D. (1988). Bingeing behavior and plasma amino acids: a possible involvement of brain serotonin in bulimia nervosa. Psychiatry Research, 23, 31-43.

Lacey, J. H. and Evans, C. (1986). The impulsivist: A multi-impulsive personality disorder. British Journal of Addictions, 81(5), 641-649.

Lacey, J. H. and Moureli, E. (1986). Bulimic alcoholics: Some features of a clinical subgroup. Br 7 Addictions, 81, 389-393.

Logan, G. D. and Cowan, W. B. (1984). On the ability to inhibit thought and action: A theory of an act of control. Psychological Review, 91, 295-327.

Lucas, A. R., Beard, C. M., O'Fallon, W. M. and Kurland, L. T. (1988). Anorexia nervosa in Rochester, Minnesota: A 45-year study. Mayo Clinic Proceedings, 63(May), 433-442.

Murphy, D. L., Zohar, J., Benkelfat, Z., Pato, M. T., Pigott, T. A. and Insel, T. R. (1989). Obsessive Compulsive Disorder as a 5-HT subsystem related behavioural disorder. British Fournal of Psychiatry, 155(Suppl. no. 8), 15-24.

Neely Wold, P. (1983). Anorexic syndromes and affective disorder. The Psychiatric Journal of the University of Ottawa, 8, 116-119.

Nielsen, S. (1990). The epidemiology of anorexia nervosa in Denmark from 1973 to 1987: A nationwide register study of psychiatric admission. Acta Psychiatrica Scandinavica, 81, 507514.

Norring, C., Sohlberg, S., Rosmark, B., Humble, K., Holmgren, S. and Nordquist, C. (1989). Ego functioning in eating disorders: Description and relation to diagnostic classification. International Journal of Eating Disorders, 8(6), 607-621.

Piran, N., Lerner, P., Garfinkel, P. E., Kennedy, S. H. and Brouillette, C. (1988). Personality disorders in anorexic patients. International Journal of Eating Disorders, 7(5), 589-599.

Pyle, R. L., Mitchell, J. E. and Eckert, E. D. (1981). Bulimia: a report of 34 cases. Fournal of Clinical Psychiatry, 42, 60-64.

Rothenberg, A. (1990). Adolescence and eating disorder: The obsessive-compulsive syndrome. Psychiatric Clinics of North America, 13(3), 469-488.

Russell, G. F. M. (1979). Bulimia nervosa: An ominous variant of anorexia nervosa. Psychological Medicine, 9, 429-448.

Sanfuentes, M. T. and Lolas, F. (1988). Eating behavior and personality: An exploratory analysis. Personality and Individual Differences, 9(2), 435-437.

Schachar, R. and Logan, G. D. (1990). Impulsivity and inhibitory control in normal development and childhood psychopathology. Developmental Psychology, 26(5), 710-720.

Schalling, D., Edman, G., Åsberg, M. and Oreland, L. (1988). Platelet MAO activity associated with impulsivity and aggressivity. Personality and Individual Differences, 9(3), $597-605$.

Shaye, R. (1989). Relationships between impulsivity and eating behaviour under varying 
conditions of stress and food deprivation. Personality and Individual Differences, 10(7), 805808.

Sohlberg, S., Norring, C., Holmgren, S. and Rosmark, B. (1989). Impulsivity and the longterm prognosis of psychiatric patients with anorexia nervosa/bulimia nervosa. Fournal of Nervous and Mental Disorders, 177(5), 249-258.

Sohlberg, S., Norring, C. and Rosmark, B. (in press). Prediction of the course of anorexia nervosa/bulimia nervosa over three years. International Fournal of Eating Disorders.

Soubrié, P. (1986). Reconciling the role of central serotonin neurons in human and animal behavior. Behavioral and Brain Sciences, 9(2), 319-364.

Southwick, L. and Steele, C. M. (1987). Restrained drinking: Personality correlates of a control style. Fournal of Drug Issues, 17(4), 349-358.

Strober, M. (1981). The significance of bulimia in juvenile anorexia nervosa; An exploration of possible etiological factors. International Journal of Eating Disorders, 1(1), 28-43.

Strober, M. (1984). Stressful life events associated with bulimia in anorexia nervosa: Empirical findings and theoretical speculations. International fournal of Eating Disorders, 3(2), 3-16.

Strober, M. (1990). Opening remarks, Plenary session on "Course and outcome predictors in eating disorders". Fourth International Conference on Eating Disorders. April 27-29. New York, USA.

Strober, M. and Humphrey, L. (1987). Familial contributions to the etiology and course of anorexia nervosa and bulimia. Fournal of Consulting and Clinical Psychology, 55(5), 654-659.

Strober, M. (in press). Disorders of the self in anorexia nervosa: An organismic-developmental paradigm. In "Psychodynamic Treatment of Anorexia Nervosa and Bulimia" (Ed. C. Johnson) Guilford Press, New York.

Swift, W. J., Bushnell, N. J., Hanson, P. and Logemann, T. (1986). Self-concept in Anorexia nervosa. Fournal of the American Academy of Child Psychiatry, 25(6), 826-835.

Toner, B. B., Garfinkel, P. E. and Garner, D. M. (1987). Cognitive style of patients with bulimic and diet-restricting anorexia nervosa. American Fournal of Psychiatry, 144(4), 510512.

Vandereycken, W. and Vanderlinden, J. (1983). Denial of illness and the use of selfreporting measures in anorexia. International Journal of Eating Disorders, 2(4), 101-107.

Wardle, J. and Marsland, L. (1990). Adolescent concerns about weight and eating: A socialdevelopmental perspective. Fournal of Psychosomatic Research, 34(4), 377-391.

Woell, C., Fichter, M. M., Pirke, K.-M. and Wolfram, G. (1989). Eating behavior of patients with bulimia nervosa. International fournal of Eating Disorders, 8(5), 557-568.

Wonderlich, S. A., Swift, W. J., Slotnick, H. B. and Goodman, S. (1990). DSM-III-R personality disorders in eating-disorder subtypes. International Fournal of Eating Disorders, 9(6), 607-616.

Woznica, J. G. (1990). Delay of gratification in bulimic and restricting anorexia nervosa patients. Fournal of Clinical Psychology, 46(6), 706-712. 


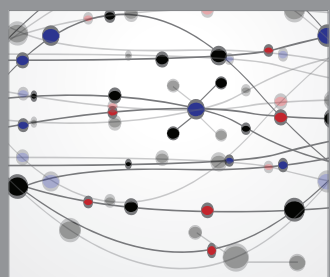

The Scientific World Journal
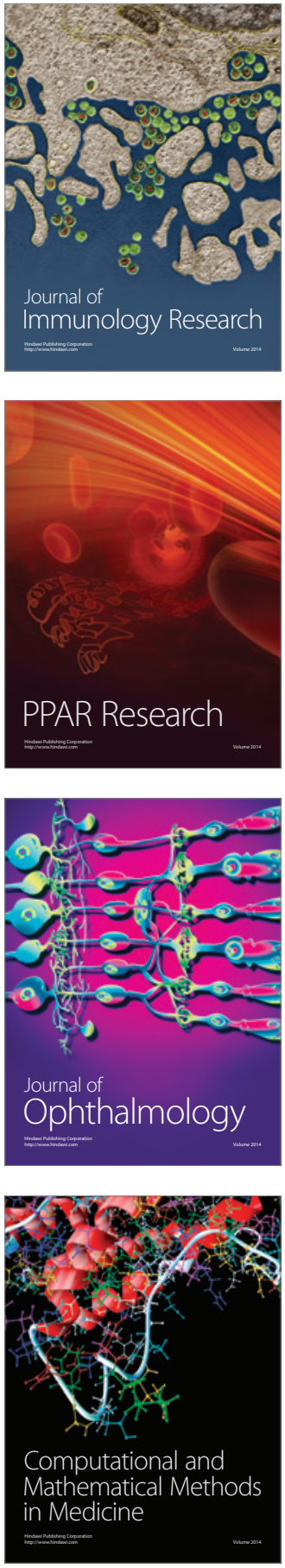

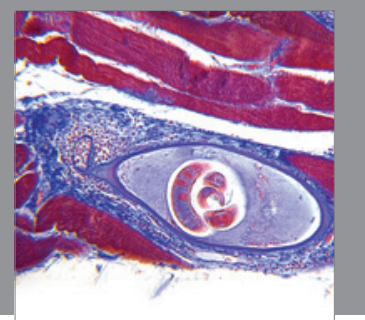

Gastroenterology

Research and Practice
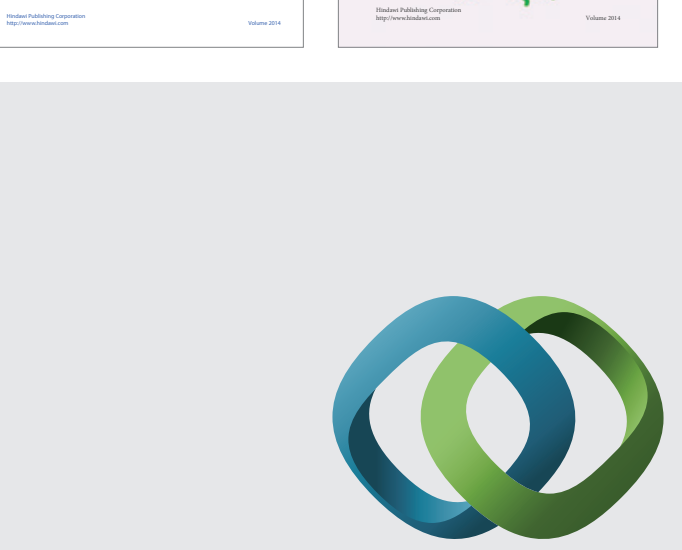

\section{Hindawi}

Submit your manuscripts at

http://www.hindawi.com
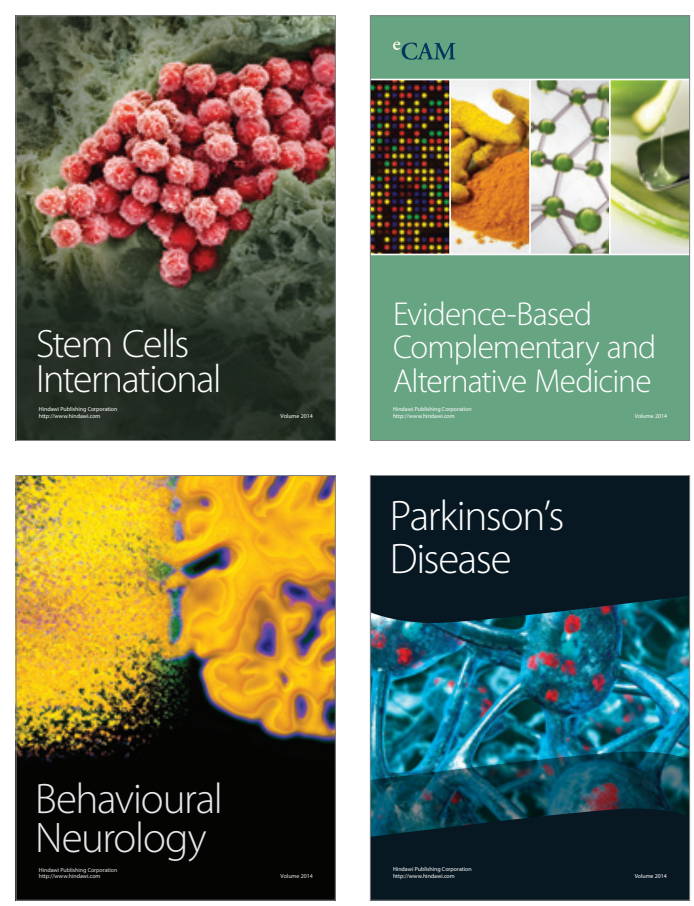

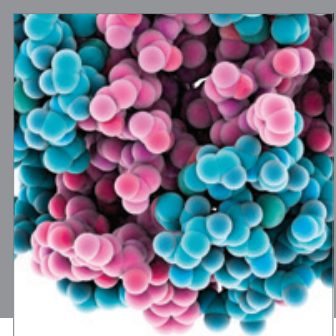

Journal of
Diabetes Research

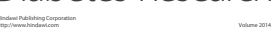

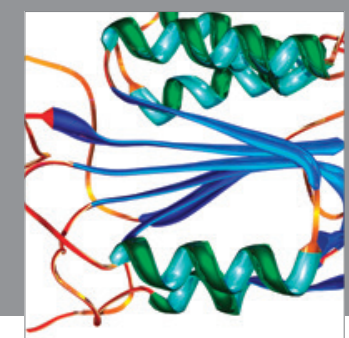

Disease Markers
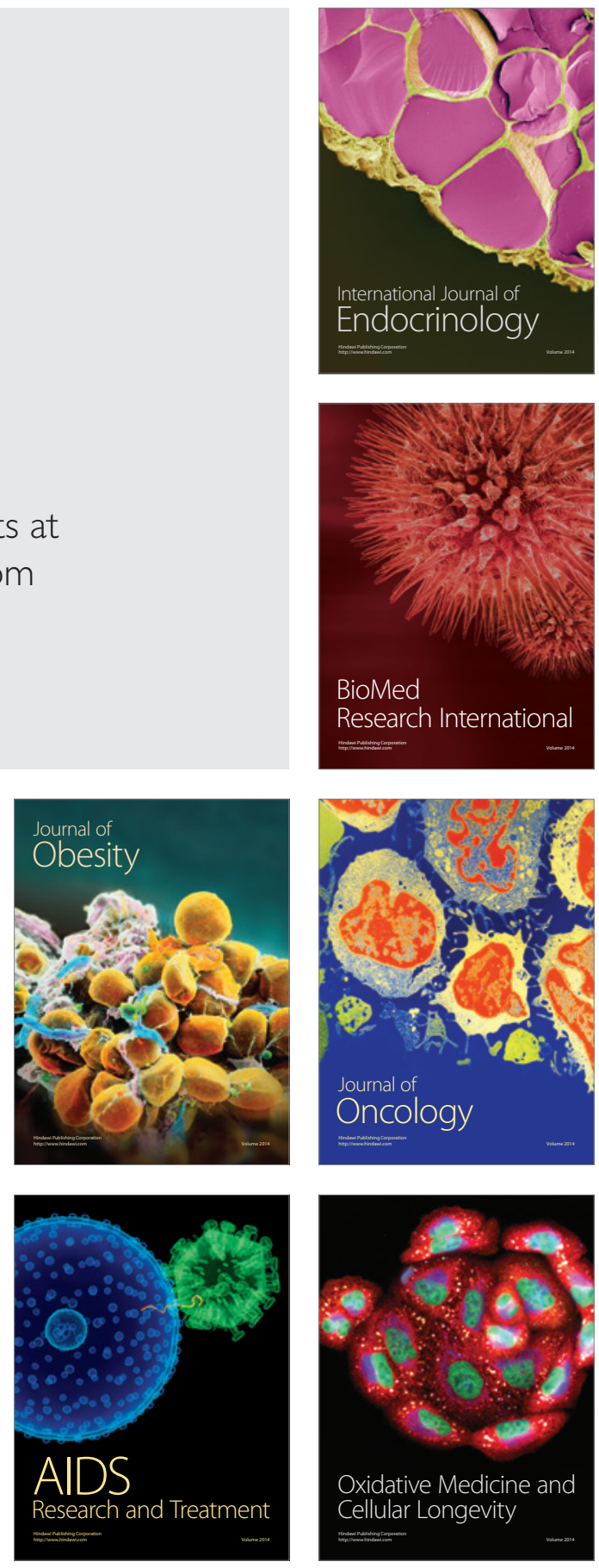